\title{
Desde qué versiones epistemológicas construyen modelos mentales los profesores en formación inicial: una investigación didáctica
}

\author{
Rafael Yecid Amador Rodríguez ${ }^{1}$ \\ Rómulo Gallego Badillo \\ Royman Pérez Miranda ${ }^{2}$
}

Artículo recibido: 9-10-2007 y aprobado: 27-11-2008

On what epistemological versions initial training science teachers build mental models: a didactical research

Resumen: En este artículo se presentan los resultados obtenidos en un trabajo de investigación didáctica con profesores en formación inicial en química de la Universidad Pedagógica Nacional, Bogotá, Colombia, en 2005, su objeto fue identificar y caracterizar los modelos mentales con los cuales ingresaron al proceso y las modificaciones que fueron consecuencia del trabajo didáctico realizado con ellos.

Palabras clave: Modelos mentales, modelo científico, formación de profesores, didáctica de las ciencias, modelo del flogisto y modelo de la oxidación.
Abstract: This paper presents the achieved results of a didactical research work with initial training chemistry teachers from Universidad Pedagógica Nacional in Bogotá, Colombia, during 2005, which goal was the identification and characterization of the mental models before the process and the modifications caused by the didactical activities.

Key words: Mental models, scientific model, teachers' training, science education, phlogiston model and oxidation model.

1 Profesor Universidad Distrital Francisco José de Caldas. Director Centro de Investigaciones Fundación de Educación Superior, Insutec, Bogotá, Colombia. Grupo de Investigación: Representaciones y Conceptos Científicos.

Correo electrónico: rafaelyecid@gmail.com

2 Profesores de la Universidad Pedagógica Nacional. Correo electrónico: rgallego@pedagogica.edu.co, centro. investigacion@insutec.edu.co y royman@pedagogica.edu.co

8 TE $\Delta$ N. $^{\circ} 24 \cdot$ Segundo semestre de $2008 \cdot$ pp. 8-22 


\section{Introducción}

La investigación se adelantó con estudiantes que optaron por formarse como profesores de química en el programa de Licenciatura en Química de la Universidad Pedagógica Nacional, durante el primer semestre de 2005, e inscritos en el espacio curricular denominado Teorías Químicas I, el número total fue de treinta y dos estudiantes.

El objetivo del trabajo fue identificar y caracterizar los modelos mentales con respecto al fenómeno de combustión para precisar si esos modelos mentales se aproximaron o no al modelo de Stahl o al de Lavoisier o de sentido común. Además, se buscaba establecer las modificaciones introducidas luego de un trabajo didáctico fundado en la lectura, elaboración de composiciones y discusión en el aula de las interpretaciones que dieron a la propuesta de Stahl y Lavoisier.

La investigación se inscribió en los actuales presupuestos de la didáctica de las ciencias y específicamente en la formación inicial de profesores de ciencias en la perspectiva de un cambio en las concepciones histórica-epistemológica previamente elaboradas como modelos mentales de sentido común. Se aproximó el trabajo a lo que en la actualidad realizan los especialistas: la reconstrucción y construcción de modelos científicos en el aula o de la didáctica de la modelación.

\section{Presupuestos conceptuales}

\section{La categoría de modelo científico}

Los presupuestos teóricos que orientaron la investigación parten de una versión epistemológica constructivista. Desde tal formulación, se asume que el conocimiento científico es una construcción social (Hodson, 1992); desde esta afirmación se considera que el desarrollo de las ciencias se puede explicar a partir de la propuesta de la existencia, en cada época, de teorías rivales (Popper, 1962), de paradigmas en competencia (Kuhn, 1972) o de programas de investigación que compiten entre sí (Lakatos, 1983). Así, se daría cuenta de esa historia en términos de sustitución de teorías, de cambio de paradigmas o de abandono de programas de investigación científica.

Los investigadores en filosofía de las ciencias de la naturaleza, para abordar e interpretar la dinámica de las ciencias, tomaron como base los cambios ocurridos en el seno de la física, a comienzos del siglo XX. Para ello, acudieron a la categoría epistemológica de la teoría, propia de esta ciencia y, cuya estructura proviene de la "Principia" y la "Óptica" de Newton. La comunidad científica ha propuesto que tal estructuración creó una versión "dura" de teoría, organizada en definiciones, postulados, demostraciones matemáticas y corolarios. Por tal motivo, se ha retomado la categoría epistemológica de modelo científico.

En los últimos años, los filósofos e historiadores de las ciencias concuerdan en que los planteamientos anteriormente enunciados poseen rigor conceptual y metodológico, por lo que sustentan el desarrollo de la física como ciencia (Campanario, 2004). Por tal razón, para interpretar el desarrollo de otras ciencias de la naturaleza como biología, geología y química, se acude a la categoría epistemológica del modelo científico.

$\mathrm{Al}$ respecto, parece haber consenso entre los especialistas en cuanto a lo que se quiere significar con esta categoría. 
Así, cada comunidad conciben los modelos científicos como representaciones idealizadas de eventos físicos en la naturaleza, construidos por hombres y mujeres de ciencias ante la necesidad de comprender los fenómenos de la naturaleza, formular y resolver problemas sobre éstos. La formulación de todo modelo se inscribe en una tradición comunitaria de producción de conocimiento propia de cada una de las ciencias de la naturaleza, todo modelo científico es una estructura conceptual y metodológica (Islas y Pesa, 2004) expresada en el lenguaje especializado que es característico de la época histórica en la que se propone y se admite el modelo.

Los modelos científicos son propuestas teóricas utilizadas como herramientas auxiliares de representación, para explicar, predecir y transformar el mundo. Los modelos científicos son construcciones provisionales y perfectibles; es decir, ningún modelo científico proporciona una explicación verdadera y absoluta (Galogovsky y Adúriz-Bravo, 2001).

\section{El concepto de modelación en la enseñanza de las ciencias de la naturaleza}

Izquierdo y Adúriz-Bravo (2005) consideran la "modelización científica" como aquel proceso de transformación del mundo que se produce como consecuencia del pensamiento científico. Partiendo de dicha afirmación, los autores de este documento consideran que en el sistema aula se presentan dos momentos de modelación, uno generado por el docente y otro por los estudiantes. Como se ha expuesto, los investigadores de cada disciplina han realizado una modelación del mundo físico, construyendo modelos mentales con la intención de interpretarlo; dichos modelos se transforman en modelos científicos por lo que son avalados y acogidos por la comunidad científica. Partimos de la idea de que en el sistema aula el conocimiento científico (modelos científicos) se hace objeto de trabajo por parte del didacta de las ciencias, buscando que los estudiantes construyan sus explicaciones de los fenómenos desde sus modelos mentales. Pensar que el conocimiento científico se hace objeto de trabajo en el aula es considerar que el docente le atribuye el carácter de enseñable.

¿Quién modela el conocimiento científico en el sistema aula?: el docente, en principio, ya que presenta su versión de los consensos obtenidos por la comunidad científica en cuanto a explicaciones de los fenómenos. Los otros integrantes del sistema aula son los estudiantes; ellos, en el momento que el didacta presenta su versión acerca de los consensos de la comunidad científica, la analizan e interpretan desde sus estructuras mentales y las modifican de acuerdo con su criterio. El proceso mediante el cual los estudiantes elaboran sus reconstrucciones o construcciones de sus estructuras mentales modelan el conocimiento científico que el profesor ha hecho objeto de trabajo en el aula, proceso en el que genera para sí un modelo mental explicativo de ese conocimiento, modelo mental que se puede categorizar epistemológicamente.

Lo anotado se presentaría en caso que, como es de esperarse, el profesor asuma su trabajo profesional en la didáctica de la modelación, ya que no podría ser así, si sigue el paradigma de la 
transmisión verbal y la repetición memorística de contenidos curriculares, esto último posiblemente desde una posición histórica-epistemológica de la ciencias de carácter empiríco-positivista.

Es necesario aclarar que se acude a la denominación de sistema aula en razón de sus protagonistas, el profesor y los estudiantes, el modelo científico que se hace objeto de trabajo en el aula y las interacciones profesor-estudiante, el profesor-modelo científico, estudiantemodelo científico y estudiante-estudiante, interacciones de carácter cognoscitivo y no lineal, es decir, no siguen la relación simple entre causa-efecto.

\section{De los modelos mentales: una posición conceptual}

Se ha conceptualizado que los estudiantes construyen modelos mentales para dar cuenta de los fenómenos que acontecen en la naturaleza; esto es, representación "cognoscitiva interna" que ellos elaboran para formularse una posible explicación de un evento del mundo físico (Gutiérrez, 2005).

Los modelos mentales son representaciones que las personas elaboran idiosincrásicamente con el objeto de representar aquellos eventos que suceden en la naturaleza o, como lo denominan estos investigadores, "estados de cosas más abstractos” (Moreira y Rodríguez Palmero, 2002). En consecuencia, en esta investigación se asumió que los modelos mentales pueden tomarse como representaciones cognoscitivas internas de carácter conceptual, metodológico, actitudinal y axiológico (Gallego, Pérez, Torres de Gallego y Amador-Rodríguez, 2004) desde las cuales los individuos reconstruyen y construyen explicaciones desde sí, para sí y con los demás.

Es de anotar que algunos investigadores en el campo de la filosofía de las ciencias delimitan los presupuestos del constructivismo y del empiropositivismo como versiones epistemológicas soslayan las interacciones sujeto-objeto, así como sus correlaciones contextuales con la sociedad en la que se proponen las teorías o modelos científicos (Giere, 1992).

Para obviar las objeciones anotadas, los estudiosos acuden a lo que se ha denominado realismo científico, visión que encuentra su sustento en el campo del naturalismo científico, versión epistemológica que, al dar entrada a componentes como la psicología y la sociología de las ciencias, permite justificar tanto las decisiones de los científicos como sus intereses y, desde ese conjunto, formular sus teorías o modelos como explicaciones de eventos que suceden en la naturaleza. Sostienen los investigadores del naturalismo científico que la tesis central de esta posición estriba en que la ciencia debe ser estudiada de igual manera que cualquier otro fenómeno empírico o natural (Zamora Bonilla, 2000).

De acuerdo con el párrafo anterior, en la Figura 1, se presenta un esquema que muestra los diferentes modelos mentales explicativos que construyen los individuos a partir de unos escenarios establecidos. Dicho modelo teórico fue construido con la intención de generar criterios de análisis sobre las composiciones que elaboraron los profesores en formación inicial en química en cuanto al concepto de combustión. 
Figura 1: Modelo teórico para la comprensión de modelos mentales explicativos.

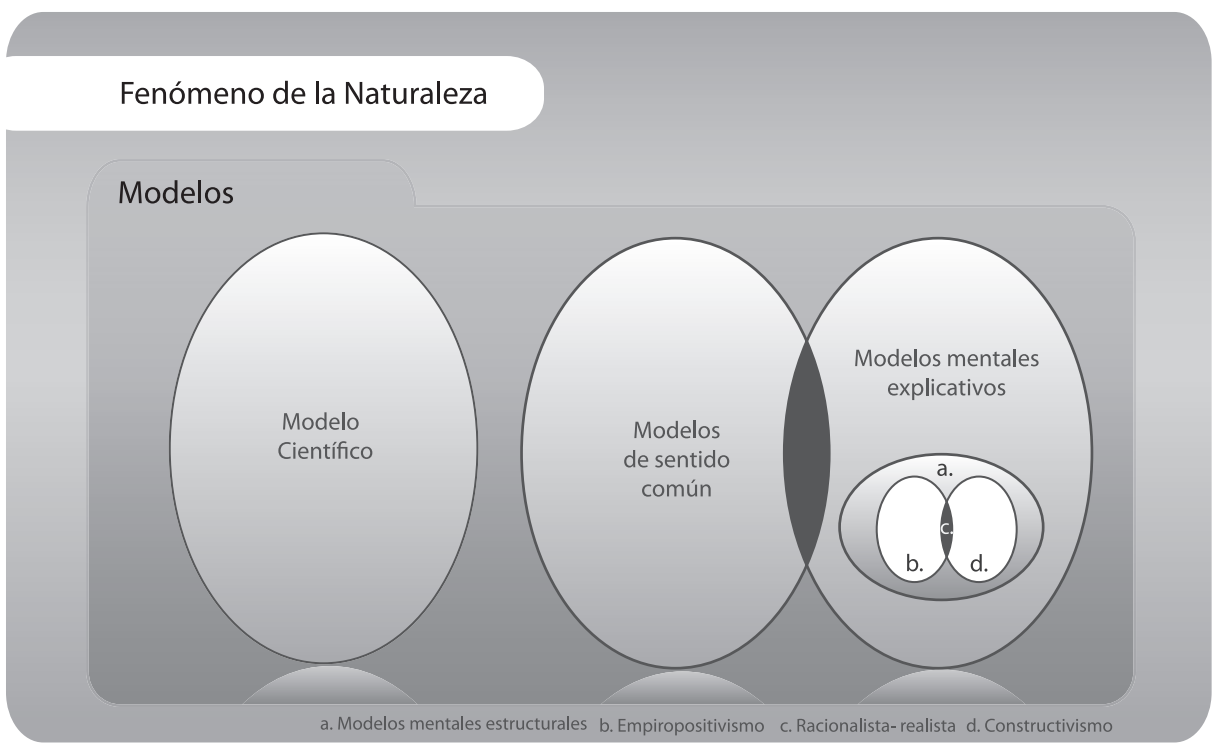

Fuente: Amador-Rodríguez, 2006.

Lo anterior, resume la idea de que existe una realidad extrasubjetiva constituida por los aconteceres que el sujeto cognoscente, individual y colectivo, transforma desde su experiencia de construcción de conocimiento en fenómeno de la naturaleza, esto es, hace una lectura de esos aconteceres. La lectura supone la inscripción en un modelo científico previo o en la propuesta de uno nuevo, modelos mentales explicativos y estructurantes que pueden estar fundamentados en una versión epistemológica de carácter empiropositivista, racionalista o constructivista.

En este mismo orden de ideas, se reconoce que la gente del común también construye modelos acerca de los fenómenos de la naturaleza, modelos que por supuesto son explicativos y estructurantes, elaborados a partir del saber común y cotidiano a los que se les podría atribuir una aproximación empirista de secano o ingenua.

Como se propone en el modelo teórico (Figura 1), los modelos mentales explicativos que elaboran los individuos en general, y los profesores en formación, en especial, son representaciones que se construyen para dar cuenta de un suceso físico. Se tomó la decisión de llamarlos modelos mentales explicativos porque no sólo son representaciones internas que explican el evento, sino que se fundamentan en el proceso discursivo que permite comunicar y contrastar con el otro, el producto de la construcción de saber sobre el particular. Los modelos mentales explicativos son clasificados en modelos de sentido común y modelos mentales estructurantes. Estos últimos se pueden diferenciar a partir de tres versiones epistemológicas (Amador-Rodríguez, Gallego Badillo y Pérez Miranda, 2005a y 2005b). ¿Qué son los modelos 
de sentido común?, se piensa que son aquellos que elaboran las personas a partir de su relación e interacción con el mundo sin referirlos a una educación escolarizada. Estos modelos no poseen fronteras bien definidas, no son científicos, es decir, surgen de las creencias de las personas con el objeto de interpretar el mundo que las rodea.

Se propone que los modelos mentales estructurantes son aquellos que elabora el individuo o profesor en formación a partir de una intervención didáctica, esto es, son los modelos de sentido común modificados mediante un trabajo didáctico. Se considera que tal construcción o reconstrucción se realiza en la medida en que los profesores en formación trabajan a partir de las propuestas de quienes han formulado modelos científicos.

\section{Metodología}

Para la investigación, los profesores en formación trabajaron con los modelos que formularon Georg Ernst Stahl y Antoine Laurent Lavoisier sobre el fenómeno de la combustión. Un interrogante que surgió en el transcurso de la investigación es si los modelos mentales estructurantes que elaboran los profesores en formación se pueden diferenciar unos de otros; para ello se recurrió a tres versiones epistemológicas: el empiropositivismo, el constructivismo y el de racionalidad-realidad.

Se considera que desde estas versiones se puede identificar: si los modelos mentales explicativos que construyen y reconstruyen los profesores en formación, se acercan a la dinámica del desarrollo del conocimiento científico.
La investigación se adelantó con treinta y dos (32) estudiantes del primer semestre de 2005 que han optado por ser Licenciados en Química de la Universidad Pedagógica Nacional. El estudio se adelantó durante cuatro semanas en el espacio académico denominado Teorías Químicas I, siendo el Profesor Royman Pérez Miranda titular y responsable de dicho espacio.

\section{Estrategia didáctica desarrollada con profesores en formación inicial}

El trabajo de investigación tuvo dos momentos: primero, determinar la versión epistemológica desde la cual los profesores de la Universidad Pedagógica Nacional en formación inicial en química reconstruyen y construyen una versión de la química como ciencia. Y, segundo, descubrir si, a partir de un trabajo didáctico- en el cual se estudiaron dos etapas históricas importantes en la química, (propuestas de Stahl y Lavoisier en lo que se refiere a la interpretación del fenómeno de la combustión)- la versión de la química como ciencia se modifica y si los modelos mentales de los profesores en formación inicial se aproximan en cuanto al concepto de la combustión a las propuestas de la comunidad científica.

Planteado el marco conceptual y metodológico, se explicita a continuación la estrategia didáctica que se implementó para la investigación:

- Solución de una prueba tipo Likert. Una prueba tipo Likert se diseña con el objeto de auscultar tendencias histórico-epistemológicas y didácticas; son escalas sumatorias en las cuales el valor asignado a cada persona se obtiene mediante la suma promedio de la puntuaciones dadas a las respectivas 
proposiciones (Gallego Badillo y Pérez Miranda, 2002).

- Lectura de los documentos que presentan menos deformación conceptual de la propuesta de Georg Ernst Stahl para el concepto de combustión por parte de los profesores en formación inicial, en lo que respecta al modelo del flogisto ${ }^{1}$. Para abordar este punto en la investigación, se tomaron como referentes:

- Elaboración de composiciones por parte de los estudiantes, trabajo individual.

- Discusión con el colectivo aula en torno a la propuesta de Georg Ernst Stahl.

- Elaboración de composiciones por parte de los estudiantes, trabajo grupal.

- Lectura de los documentos que presentan más conformidad con la propuesta de Antoine Laurent Lavoisier.

- Elaboración de composiciones por parte de los estudiantes, trabajo individual.

- Discusión en el colectivo aula en torno a la propuesta de Antoine Laurent Lavoisier.

- Elaboración de composiciones por parte de los estudiantes, trabajo grupal.

- Solución de una prueba tipo Likert.

\section{Resultados y análisis de la investigación}

A continuación, se presentan los resultados obtenidos a partir de la solución de la prueba tipo Likert que los profesores resolvieron tanto al inicio de la investigación como al final de la misma y sus respectivos análisis. Por otra parte, se presentan los análisis realizados sobre las composiciones individuales y grupales que elaboraron los profesores en formación y de los conversatorios que

1 Para este punto de la investigación, se tuvieron como algunos de los referentes bibliográficos: Lavoisier, L. L. (1777), Lavoisier, L. L. (1965) y Priestley, J. (1733-1804). se adelantaron con el profesorado en formación inicial.

Fundamentos epistemológicos del profesorado en formación

Los resultados y análisis que presentamos a continuación se derivan de la prueba tipo Likert (Anexo 1) que los profesores en formación respondieron tanto al inicio como al final del proceso de enseñanza-aprendizaje. Se tomó la decisión de determinar si las tendencias epistemológicas con las que ingresan al curso de Teorías Químicas I los profesores en formación se aproximan a dos tendencias epistemológicas extremas: la versión empiropositivista y la constructivista.

En lo que hace relación a las afirmaciones que se diseñaron desde una versión constructivista, en lo que respecta al desarrollo del conocimiento químico y de la didáctica de las ciencias de la naturaleza, el $60 \%$ de los profesores no se aproxima a los presupuestos de dicha versión epistemológica, mientras que el $40 \%$ de los profesores en formación inicial presenta cambios al pensar que el conocimiento químico se ha construido en comunidad, alejándose de la idea lineal y acumulativa del desarrollo del conocimiento científico y pensando que tal dinámica es compleja y no lineal.

\section{Modelos mentales explicativos de los profesores en formación inicial en química}

Leídas las composiciones, se procedió a establecer desde qué modelo mental explicativo se elaboran las mismas; a continuación se presentan los resultados. 


\section{Criterios de análisis}

\section{Composiciones individuales y grupales}

Para analizar las composiciones efectuadas por los profesores en formación inicial en química, se partió del esquema que se expuso en este documento (Figura 1 ), en el cual se plantea que los profesores en formación elaboran modelos mentales explicativos a eventos que acontecen en la naturaleza (AmadorRodríguez, 2006); dichos modelos se pueden clasificar de la siguiente manera: modelos de sentido común y modelos mentales estructurantes; éstos a su vez se diferencian según versiones epistemológicas como el empiropositivismo, el constructivismo y el de racionalidad y realidad.

La interpretación de las composiciones elaboradas por los profesores en formación - y desde el marco conceptual que ya se expuso - obedeció a los criterios de categorización propuestos. Se encontraron afirmaciones como:

[...] El flogisto es un elemento inasible que en el momento de la combustión de un cuerpo rompe unión con éste y da paso al cambio de las propiedades del cuerpo quemado [...].

Por tanto, se ubica en un modelo mental explicativo de sentido común.

[...] Con estas propuestas tan superficiales del siglo XVII, Boyle introduce el método científico y da algunas leyes como la de la atracción o afinidad y empezó a trabajar con los gases, para dar paso a la teoría del flogisto sustentada por Sthal [...].

Lo enunciado permite una interpretación como un modelo mental estructurante desde los presupuestos empiropositivistas.
[...] En 1783 hubo un problema con la llegada del agua a ciertas ciudades de Francia. [...] Lavoisier decidió leer todo lo escrito acerca de los gases. [...] Lo más importante de Lavoisier era su actitud respecto a las teorías planteadas por el flogisto [...].

La cláusula en cuestión permite deducir su pertenencia a un modelo mental estructurante de carácter real-racional.

[...] Lavoisier puso manos a la obra y comienza a resolver el problema más importante, la participación del aire en algunas reacciones químicas. [...] Lavoisier interpretó que la transformación de los metales era a causa de la interacción con el aire puro [...].

La cita, da para pensar en un modelo mental estructurante de carácter constructivista.

\section{Modelos mentales explicativos individuales del modelo del flogisto}

Para obtener estos resultados, se realizó una lectura detallada de cada composición, ubicándolas en una de las categorías que para esta investigación se propusieron se estableció el porcentaje correspondiente para cada modelo.

Se encontró que el 24,3\% (Tabla 1) del profesorado en formación elaboró composiciones desde el sentido común- ésto es, las composiciones no se acercan a lo planteado por la comunidad científicaen lo que se refiere a la construcción del concepto de combustión. Es de anotar que las composiciones se elaboraron después de que los profesores en formación inicial leyeron la propuesta que hace referencia al Modelo del flogisto.

El 51,3\% de los profesores en formación inicial escribió sus composiciones desde los presupuestos empiropositivistas. 


\begin{tabular}{|c|c|c|c|}
\hline \multicolumn{4}{|c|}{ Modelos mentales explicativos del Modelo del Flogisto } \\
\hline De sentido común & $24,3 \%$ & & \multirow{4}{*}{$\begin{array}{l}\text { Composiciones de los profesores en } \\
\text { formación inicial en química } \\
\text { (individual). }\end{array}$} \\
\hline Empiropositivismo & $51,3 \%$ & & \\
\hline Realidad-racionalidad & $5,5 \%$ & & \\
\hline Constructivismo & & $18,9 \%$ & \\
\hline
\end{tabular}

Tabla 1: Resultados porcentuales de las composiciones individuales: Modelo del Flogisto

Un porcentaje del $5,5 \%$ de las composiciones individuales se ubica en la versión epistemológica de racionalidadrealidad, lo que nos conduce a pensar que los profesores en formación inicial consideran que el desarrollo del conocimiento científico obedece a una interacción entre la realidad y los procesos mentales que realizan los científicos con el fin de elaborar modelos teóricos.

El 18,9\% de los profesores en formación inicial en química elaboró sus composiciones desde los presupuestos constructivistas, esto es, la tarea de los hombres y mujeres de ciencias es elaborar teorías o modelos científicos con el objeto de dar cuenta de los eventos que suceden en la naturaleza.

\section{Modelos mentales explicativos del modelo del flogisto: trabajo en colectivo}

Efectuada la socialización, el porcentaje de las composiciones elaboradas desde una versión epistemológica empiropositivista se incrementa al 75\%, el cual representa los acuerdos obtenidos por los grupos de trabajo conformados en el sistema aula. La mayoría de los grupos considera, al parecer, que el desarrollo del conocimiento científico se construye de manera lineal y acumulativa.

Realizada la socialización con el colectivo de profesores en formación en química, se detectó que las composiciones grupales no se realizaron desde la categoría de modelo de sentido común; se considera que dichos profesores comienzan a tener presente la construcción del conocimiento desde su historia, acercándose así a los consensos de la comunidad científica, en especial, a lo propuesto por Georg Ernst Stahl.

Adelantada la socialización, el porcentaje referido a la versión de racionalidad-realidad es cero. Se considera que este resultado obedece a que los documentos suministrados para el estudio del Modelo del flogisto no proporcionan una visión lo suficientemente amplia de los procesos de análisis y síntesis que efectúan los individuos de ciencias para otorgarle validez a los fenómenos naturaleza.

\begin{tabular}{lccc}
\hline & Modelos mentales explicativos del Modelo del Flogisto \\
\cline { 1 - 2 } De sentido común & $0 \%$ & & $\begin{array}{c}\text { Composiciones de los profesores en } \\
\text { formación inicial en química (grupal). }\end{array}$ \\
\cline { 1 - 2 } Empiropositivismo & $75 \%$ & $0 \%$ & $25 \%$
\end{tabular}

Tabla 2: Resultados porcentuales de las composiciones grupales: Modelo del Flogisto. 
El 25\% de las composiciones grupales considera que la versión epistemológica que sustenta la construcción del Modelo del flogisto es la constructivista, dados sus presupuestos conceptuales y metodológicos del desarrollo científico.

\section{Modelos mentales explicativos individuales del modelo de la oxidación}

\begin{tabular}{|c|c|c|c|c|}
\hline \multicolumn{5}{|c|}{ Modelos mentales explicativos del Modelo de la Oxidación } \\
\hline De sentido común & $6 \%$ & & & \multirow{4}{*}{$\begin{array}{l}\text { Composiciones de los profesores } \\
\text { en formación inicial en química } \\
\text { (individual). }\end{array}$} \\
\hline Empiropositivismo & & $39,6 \%$ & & \\
\hline Realidad-racionalidad & & $9 \%$ & & \\
\hline Constructivismo & & & $45,4 \%$ & \\
\hline
\end{tabular}

Tabla 3: Resultados porcentuales de las composiciones individuales: modelo de la oxidación.

Como se presenta en la Tabla 3, aún prevalece la elaboración de composiciones individuales desde un modelo de sentido común, representado por el 6\% de los profesores en formación.

El 39,6\% de los profesores en formación inicial elaboró sus composiciones desde los presupuestos empiropositivistas, lo que lleva al convencimiento de que aún predomina la idea de un desarrollo lineal y acumulativo del conocimiento científico (ver Tabla 3).

El 9\% de los profesores en formación elaboró sus composiciones (indi- viduales) desde los presupuestos de la racionalidad-realidad como versión epistemológica del desarrollo del conocimiento científico.

Se observa que se incrementa el porcentaje de producción de composiciones individuales desde una versión constructivista en comparación con los resultados arrojados en la Tabla 1; este porcentaje es del $45,4 \%$, indicando que las concepciones de los futuros profesores acerca del concepto combustión se aproxima a lo propuesto por Lavoisier en el siglo XVIII (Tabla 3).

Modelos mentales explicativos del modelo de la oxidación: trabajo en colectivo

\begin{tabular}{|c|c|c|}
\hline \multicolumn{3}{|c|}{ Modelos mentales explicativos del Modelo de la Oxidación } \\
\hline De sentido común & $0 \%$ & \multirow{4}{*}{$\begin{array}{l}\text { Composiciones de los profesores en } \\
\text { formación inicial en química } \\
\text { (grupal) }\end{array}$} \\
\hline Empiropositivismo & $40 \%$ & \\
\hline Realidad-racionalidad & $20 \%$ & \\
\hline Constructivismo & & \\
\hline
\end{tabular}

Tabla 4: Resultados porcentuales de las composiciones grupales: modelo de la oxidación. 
Efectuada la socialización en el sistema aula y conformados los grupos de trabajo, el porcentaje representativo en lo que respecta a la elaboración de composiciones desde un conocimiento de sentido común es cero (ver Tabla 4). Dicho porcentaje hace pensar que los profesores en formación, ya no poseen un conocimiento de la ciencia o una explicación de los fenómenos físicos desde unas creencias cotidianas.

Después de la socialización en el sistema aula, el $40 \%$ de los grupos de trabajo realizó sus composiciones desde los presupuestos del empiropositivismo, confirmando así la tendencia epistemológica desde la cual algunos profesores en formación dan cuenta del desarrollo del concepto de combustión (Tabla 4).

El porcentaje de los grupos que elaboró sus trabajos bajo la versión epistemológica de racionalidad-realidad fue del 20\%. Por ello, podemos afirmar que los futuros profesores en química consideran que el desarrollo del conocimiento científico es dado a partir de un proceso de síntesis-análisis en el individuo y que, desde este razonamiento, hombres y mujeres le otorgan validez a los fenómenos naturaleza.

\section{Conversatorio con el profesorado en formación} Como ya se había dicho, este conversatorio se generó en dos momentos: uno cuando se abordó la propuesta de Stahl; para este primer momento se establecieron tres preguntas orientadoras, las cuales se hicieron con el objeto de excluir otros que no eran de prioridad para esta investigación. Las preguntas orientadoras fueron:
- ¿Los metales en el proceso de la combustión desprenden su principio inflamable (flogisto)?

- ¿El flogisto es una sustancia?

- ¿En el proceso de la combustión, se puede afirmar que los productos pesan más, ya que el flogisto presenta un peso negativo, lo que le permite al metal tener un peso inferior?

Cuando se trabajó la propuesta de Lavoisier, las preguntas orientadoras fueron:

- ¿Se puede afirmar que el flogisto es una sustancia?

- ¿Podríamos comprobar la existencia del flogisto?

- ¿Se requiere de la presencia del oxígeno para que sucedan los fenómenos de la combustión, calcinación y procesos de respiración?

Se visualizó, para esta investigación, que las preguntas le facilitaran a los profesores en formación seleccionar, cuál de los dos modelos científicos posee una mayor explicación para el fenómeno de la combustión.

Después de los conversatorios, se elaboraron las composiciones grupales, resultados que presentamos anteriormente, en los que se puede observar la manera como los modelos mentales explicativos de los profesores, se modifican.

\section{Análisis comparativo de resultados}

A continuación, se presenta una tabla en la que se da a conocer la triangulación de los análisis derivados de los resultados obtenidos de las pruebas tipo Likert, las composiciones y los conversatorios. 


\begin{tabular}{ccc}
\hline \multicolumn{1}{c}{ Cuadro comparativo } \\
\hline & \multicolumn{1}{c}{ Composiciones } \\
\hline \multirow{2}{*}{$\begin{array}{c}\text { Pruebas tipo profesores en formación otorgan importancia al estudio de la } \\
\text { historia de las ciencias. }\end{array}$} & $\begin{array}{c}\text { Se observan cambios significativos individuales en cuanto a la } \\
\text { concepción de ciencia como una construcción humana. }\end{array}$ \\
\cline { 2 - 3 } & $\frac{\text { Los cambios se manifiestan lentos y difíciles. }}{\text { Los instrumentos facilitaron la objetivación de los cambios. }}$ \\
\cline { 2 - 3 } & Abandonan la visión de las ciencias desde el sentido común. \\
\hline
\end{tabular}

Tabla 5: Triangulación de datos.

\section{Conclusiones}

La investigación que se adelantó en el espacio académico: Teorías Químicas I permitió formular una estrategia didáctica no convencional, esto es, se diseñó la estrategia desde los presupuestos constructivistas, los cuales parten del convencimiento de que la reconstrucción y construcción del conocimiento es un proceso colectivo y no individual. En la metodología de enseñanza, la historia de la química - y en específico del concepto de combustión - desempeñó un papel importante, ya que los profesores en formación se remitieron a la historia para la elaboración de sus composiciones. Se afirma que dicha estrategia fue exitosa, ya que se evidenciaron cambios en los modelos mentales explicativos.

Valga la pena aclarar, que Teorías Químicas I es un espacio académico prescrito en el programa de Licenciatura en Química, en el cual el conocimiento químico se hace objeto de trabajo y en el que la epistemología y la historia no constituyen los intereses primordiales del mismo. Vistos los análisis de los resultados - de entrada y salida de las pruebas tipo Likert - podríamos afirmar que hubo cambios, aunque no significa- tivos, en las concepciones epistemológicas y didácticas.

Se parte del convencimiento de que transformar las concepciones epistemológicas de estos futuros profesores en formación no es un proceso inmediato, ya que demanda tiempo y esfuerzo tanto de los profesores en formación como de los profesores en ejercicio.

Se encuentra que los profesores en formación inicial en química le atribuyen importancia a la historia de las ciencias en la enseñanza de las mismas, lo que se reflejó evidentemente en las composiciones. Involucrar la historia de la química - en especial la de la construcción del concepto de combustión en las composiciones elaboradas por los futuros profesores de química- les permitió conocer los problemas y las dificultades que enfrentaron quienes elaboraron el concepto de combustión $y$, por ende, acercarse a lo que la comunidad ha propuesto al respecto.

Realizado el trabajo didáctico, se asevera que los modelos mentales explicativos de los profesores en formación inicial en química se van modificando y acercándose a los consensos de la comunidad científica. Por tal motivo, 
se afirma que los modelos mentales explicativos que construyen los futuros profesores en formación no son estáticos, permitiendo concluir que el proceso de aprendizaje, además de dinámico, no es lineal ni acumulativo.

En lo que respecta a las composiciones, es de anotar que se evidencia una reconstrucción y construcción de los modelos mentales explicativos y que dichos modelos, en su gran mayoría, están soportados desde los presupuestos del constructivismo.

\section{Referencias bibliográficas}

Amador Rodríguez, R. (2006). Del modelo del flogisto al modelo de la oxidación: Una aproximación didáctica a la determinación de modelos mentales en la formación de profesores en química. Tesis de Maestría. Universidad Pedagógica Nacional.

Amador Rodríguez, R., Gallego Badillo, R. y Pérez Miranda, R. (2005a). La producción de modelos mentales explicativos en profesores en formación: Una experiencia didáctica en la enseñanza de la química. Memorias del Segundo Encuentro Internacional de Enseñanza y aprendizaje de las Ciencias y las Tecnologías. Instituto Universitario de la Paz. Barrancabermeja. Colombia.

Amador Rodríguez, R. Y, Gallego Badillo, R. y Pérez Miranda, R. (2005b). Del Flogisto a la Oxidación: la construcción de modelos explicativos en la formación inicial de profesores de química. Memorias del V Encuentro Nacional de Pesquisa em Educaçao em Ciencias. Abrapec. Bauru-Brasil.

Campanario, J. M. (2004). Científicos que cuestionan los paradigmas dominantes: Algunas implicaciones para la enseñanza de las ciencias. Revista Electrónica de Enseñanza de las Ciencias, (3)3. Extraido desde http://www. saum.uvigo.es/reec/

Galagovsky, L. y Adúriz-Bravo, A. (2001). Modelos y analogías en la enseñanza de las ciencias naturales. El concepto de modelo didáctico analógico. Enseñanza de las Ciencias, 19(2), 231-242.

Gallego Badillo, R. y Pérez Miranda. R. (2002). El problema del cambio en estudiantes de formación avanzada. Enseñanza de las Ciencias, 20(3), 401-414.

Gallego Badillo, R., Pérez Miranda, R., Torres de Gallego, L. N., y Amador Rodríguez, R. (2004). La formación inicial de profesores de ciencias en Colombia contrastación de los fundamentos. Bogotá: Universidad Pedagógica Nacional.

Giere, R. N. (1992). La explicación de la ciencia. Un acercamiento cognoscitivo. México: Concejo Nacional de Ciencia y Tecnología.

Gutiérrez, R. (2005). Polisemia actual del concepto "modelo mental". Consecuencias para la investigación didáctica. Investigações em Ensino de Ciências. (10)2. Extraído desde http:// www.if.ufrgs.br/ienci/

Hodson, D. (1992). In search of meaningful relationship: an exploration of some issues relating to integration in science and science education. International Journal of Science Education, (5)14, 541-566.

Islas, S. M. y Pesa, M. A. (2004). Concepciones de los profesores sobre el rol de los modelos científicos en clases de física. Revista de Enseñanza de la Física, (1) 17, 43-50. 
Izquierdo M. y Adúriz Bravo. A. (2005). Los modelos teóricos para la ciencia escolar. Un ejemplo de química. Enseñanza de las Ciencias, Número Extra.

Kuhn, T. S. (1972). La estructura de las revoluciones científicas. México: Fondo de Cultura Económica.

Lakatos, I. (1983). La metodología de los programas de investigación científica. Madrid: Alianza.

Lavoisier, L. L. (1965). Memorias sobre el oxígeno, el calórico y la respiración. Buenos Aires: Emecé Editores.

Lavoisier, L. L. (1777). Memoir on combustion in general. Extraído desde http://

\section{Anexos}

\section{Anexo 1}

\section{Universidad Pedagógica Nacional Facultad de Ciencia y Tecnología Departamento de Química}

Maestría en Docencia de la Química Teorías Químicas I

Prueba tipo Likert

\section{Nombre del estudiante:}

\section{Fecha:}

A continuación, usted encontrará una serie de afirmaciones numeradas de 1 a 21 , léalas cuidadosamente y en la hoja de respuestas marque una $\mathrm{X}$ en la casilla correspondiente de acuerdo con el valor que usted considere.

\section{Totalmente de acuerdo \\ 2. De acuerdo \\ 3. No sé qué decir \\ 4. En desacuerdo. \\ 5. Totalmente en desacuerdo}

\section{Universidad Pedagógica Nacional}

Facultad de Ciencia y Tecnología web.lemoyne.edu/ giunta/lavoisier1. html-

Moreira, M. A. y Rodríguez Palmero, M. L. (2002). Modelos mentales y modelos conceptuales en la enseñanza y aprendizaje de las ciencias. Revista Brasília de Pesquisa em Educação em Ciências, 2(3), 36-56.

Popper, K. (1962). La lógica de la investigación científica. Madrid: Tecnos.

Priestley, J. (1733-1804). Experiments And Observations On Different Kinds of Air. Extraído de: http://web.lemoyne. edu/ giunta/priestley.html

Zamora Bonilla, J. P. (2000). El naturalismo científico de Ronald Giere y Philip Kitcher. Revista de Filosofía, 3, 169-190.

Departamento de Química Maestría en Docencia de la Química Teorías Químicas I

1. Los químicos parten de la observación.

2. Para enseñar química basta con conocerla.

3. La química se ha desarrollado a partir de descubrimientos.

4. El papel del profesor de química es la de transmitir conocimientos.

5. La química ha sido construida por grupos de especialistas.

6. La química es un conjunto de técnicas de preparación de sustancias.

7. No hay diferencias entre las teorías de la química y las de física.

8. Aprender química es repetir lo transmitido por el profesor.

9. Para enseñar química se requiere de conocimientos didácticos.

10. La actividad inicial de los químicos es la formulación de teorías.

11.Para ser profesor de química hay que conocer la historia de esta ciencia. 
12.La didáctica de la química es una serie de técnicas de enseñanza.

13.El experimento en química permite contrastar la teoría de la que se originó.

14.La historia de la química habla de los descubrimientos realizados por los químicos.

15.Saber química es comprender cómo se construyeron sus teorías.

16.Enseñar química es explicar los contenidos de esta ciencia.

\section{Hoja de respuestas}

\section{Totalmente de acuerdo}

2. De acuerdo

3. No sé qué decir

4. En desacuerdo

5. Totalmente en desacuerdo
17.Los experimentos químicos son los anotados en las prácticas de laboratorio.

18.La química no esta conformada por teorías.

19.Para enseñar química no se requiere conocer la historia de su desarrollo.

20.Aprender química es cambiar las ideas que se han elaborado previamente de esta ciencia.

21.En la construcción histórica de la química como ciencia el método científico ha desempeñado un papel importante.

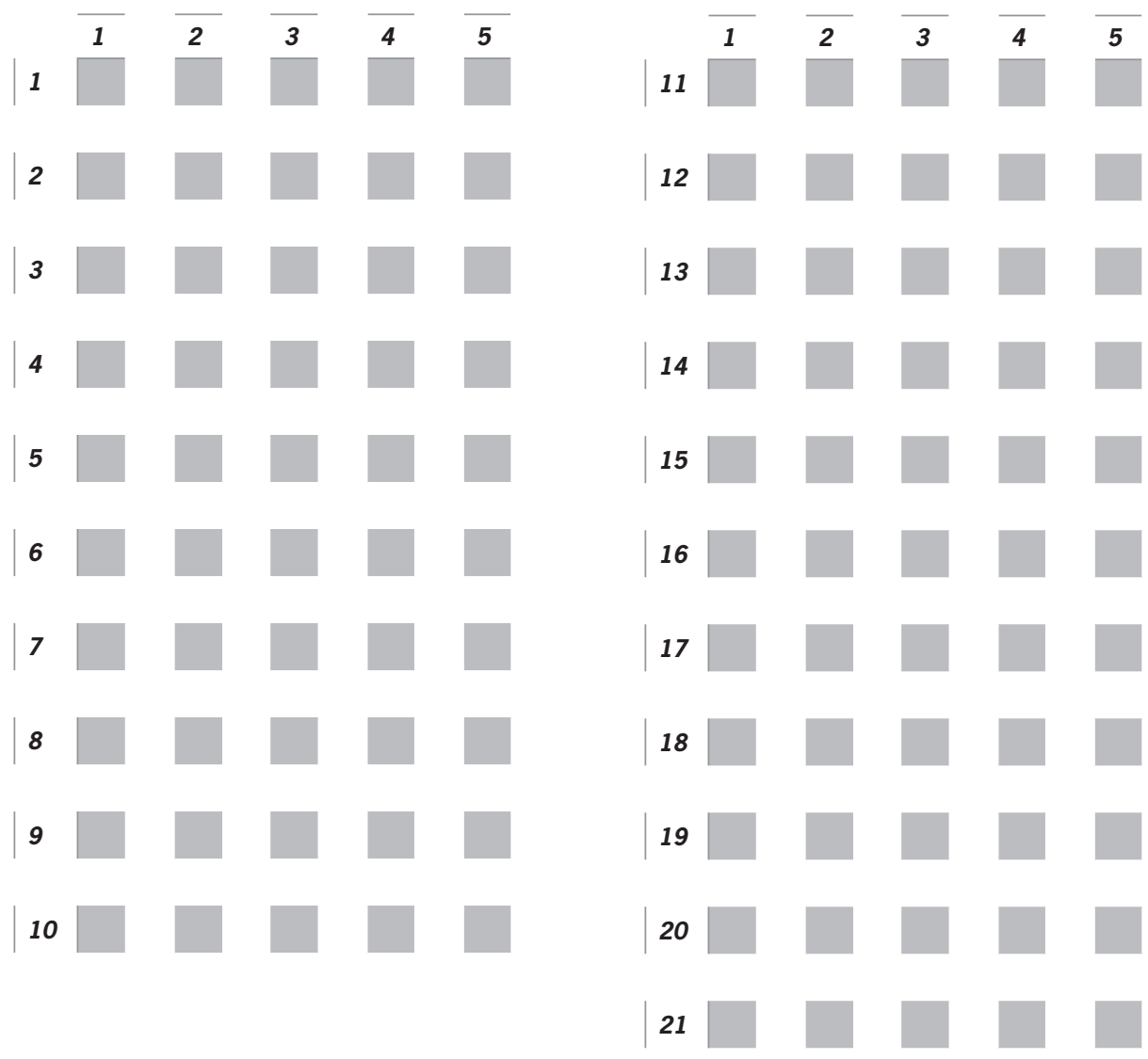

